\title{
Accurate switching intensities and length scales in quasi-phase-matched materials
}

\author{
Ole Bang, Torben Winther Graversen, and Joel F. Corney
}

Department of Informatics and Mathematical Modelling, Technical University of Denmark, DK-2800 Lyngby, Denmark

\begin{abstract}
We consider unseeded Type I second-harmonic generation in quasi-phase-matched (QPM) quadratic nonlinear materials and derive an accurate analytical expression for the evolution of the average intensity. The intensity-dependent nonlinear phase mismatch due to the QPM induced cubic nonlinearity is found. The equivalent formula for the intensity for maximum conversion, the crossing of which changes the nonlinear phase-shift of the fundamental over a period abruptly by $\pi$, corrects earlier estimates by more than a factor of 5 . We find the crystal lengths necessary to obtain an optimal flat phase versus intensity response on either side of this separatrix intensity.
\end{abstract}

Since the observation of nonlinear phase-shifts in pxcess of $\pi$ through cascading close to phase-matchingt, 2 , quadratic nonlinear or $\chi^{(2)}$ materials have been of significant interest in photonics3. With the pnaturing of the quasi-phase-matching (QPM) technique 4 , in particular by electric-field poling of ferro-electric materials, such as $\mathrm{LiNbO}_{3}$, and by quantum-well disordering in semiconductors 6 , the number of possible applications of cascading in $\chi^{(2)}$ materials has increased even more.

An effect of QPM gratings is to generate cubic nonlinearities in the equations for the average field due to non-phase-matched coupling between modest. This cubic nonlinearity appears in QPM with linear and/or nonlinear gratings 8 , it can be focusing or defocysing, depending on the sign of the phase mismatch 8 , and its strength can be significantly increased by modulating the grating 9 . Simulations of QPM systems have confirmed the presence of an intensity dependent nonlinear phase mismatch 10 and of soliton properties 7 , that can only be described by including the cubic terms. The Kerr nonlinearity is also known to distort the second-harmonic ( $\mathrm{SH}$ ) spectrum and introduce an intensity dependent ponlinear phase mismatch, as found both theoreticallyt 11 and experimentally 12. Thus, although the physical origin of the Kerr and the induced cubic nonlinearities is distinctly different, they have qualitatively similar effects on CW waves.

In this letter we focus on the intensity dependent nonlinear phase-mismatch, which implies a finite separatrix intensity that can be used in efficient all-optical switching, as was shown for the Kerr nonlinearity in poled fibres 13 and the QPM induced cubic nonlinearity 10 . A distinct feature of the averaged QPM model with induced cubic nonlinearities is that there is not a one-to-one correspondance between the physical field and the averaged field. The separatrix intensity obtained with no SH seeding in the averaged model does therefore not accurately predict the real physical separatrix intensity of unseeded second-harmonic generation (SHG). We address this discrepancy and find the exact separatrix intensity for unseeded Type I SHG in QPM samples. We further show that the averaged model gives also quantitatively inaccurate results for no seeding of the average $\mathrm{SH}$, since a basic assumption in the averaging procedure is violated, and we find the optimum crystal lengths for using the induced separatrix intensity for all-optical switching.

We consider a linearly polarized electric field $\vec{E}=$ $\hat{e}\left[E_{1}(z) \exp \left(i k_{1} z-i \omega t\right)+E_{2}(z) \exp \left(i k_{2} z-i 2 \omega t\right)+c . c.\right] / 2$, propagating in a lossless QPM $\chi^{(2)}$ medium under conditions for type I SHG. The dynamicalequations for the slowly varying envelopes take the form 14

$$
\begin{aligned}
& i d E_{1} / d z+G(z) \chi_{1} E_{1}^{*} E_{2} \mathrm{e}^{i \Delta k z}=0, \\
& i d E_{2} / d z+G(z) \chi_{2} E_{1}^{2} \mathrm{e}^{-i \Delta k z}=0,
\end{aligned}
$$

where $E_{1}(z)$ is the fundamental wave (FW) with frequency $\omega$ and wavevector $k_{1}, E_{2}(z)$ is the $\mathrm{SH}$ with wavevector $k_{2}, \Delta k=k_{2}-2 k_{1}$ is the wavevector mismatch, and $\chi_{j}=\omega d_{\text {eff }} /\left(n_{j} c\right)$, with $n_{j}=n(j \omega)$ being the refractive index and $d_{\text {eff }}=\chi^{(2)} / 2$ being in MKS units. The total intensity $I=\frac{1}{2} \eta_{0}\left(n_{1}\left|E_{1}\right|^{2}+n_{2}\left|E_{2}\right|^{2}\right)$ is conserved, where $\eta_{0}=\sqrt{\epsilon_{0} / \mu_{0}}$ is the specific admittance of vacuum. The $\chi^{(2)}$ susceptibility is modulated by the grating function $G(z)$ with unit amplitude and Fourier series $G(z)=\sigma \sum_{n} g_{n} \mathrm{e}^{i n \kappa z}$, where $g_{n}=0$ for $n$ even and $g_{n}=2 /(i \pi n)$ for $n$ odd. This zero-average square-wave modulation is typical for QPM by domain inversion in ferro-electric materials, such as $\mathrm{LiNbO}_{3}$. We define $\sigma=\operatorname{sign}(\kappa)$ so that $G(z)$ is positive in the first domain.

We further consider first order forward QPM with a short coherence length $L_{d} \sim L_{c} \ll L$, where $L$ is the crystal length, $L_{d}=\pi /|\kappa|$ is the domain length, and $L_{c}=\pi /|\Delta k|$ is the coherence length. Expanding the fields in Fourier series in the grating wavenumber $\kappa$,

$$
E_{1}=\sum_{n} w_{n}(z) \mathrm{e}^{i n \kappa z}, E_{2}=\sigma \sum_{n} v_{n}(z) \mathrm{e}^{i(n \kappa-\beta) z},
$$


where the harmonics are small compared to the df component, a simple first-order perturbation theoryd 8 gives the dynamical equations for the slowly varying (on the scale of the domain length) average field

$$
\begin{aligned}
& i d w_{0} / d z+i \rho_{1} w_{0}^{*} v_{0}+\left(\gamma_{1}\left|v_{0}\right|^{2}-\gamma_{2}\left|w_{0}\right|^{2}\right) w_{0}=0 \\
& i d v_{0} / d z+\beta v_{0}-i \rho_{2} w_{0}^{2}+2 \gamma_{2}\left|w_{0}\right|^{2} v_{0}=0
\end{aligned}
$$

where $\beta=\Delta k-\kappa \ll \kappa$ is the residual mismatch, $\rho_{j}=\chi_{j} 2 / \pi$, and $\gamma_{j}=\chi_{j} \chi_{1}\left(1-8 / \pi^{2}\right) / \kappa$. Since $L_{d} \sim L_{c} \ll L$ the sign of $\kappa$ is the sign of the mismatch, $\operatorname{sign}(\kappa)=\operatorname{sign}(\Delta k)$. Thus the QPM induced cubic nonlinearity can be both focusing and defocusing, depending on the sign of the mismatch $\Delta k$, just as the effective cubic nonlinearity obtained in the cascading limit 3 . The harmonics are given by

$$
n \kappa w_{n \neq 0}=\chi_{1} g_{n-1} w_{0}^{*} v_{0}, \quad n \kappa v_{n \neq 0}=\chi_{2} g_{n+1} w_{0}^{2},
$$

The average model (4.5) conserves the total average intensity $I_{0}=\frac{1}{2} \eta_{0}\left(n_{1}\left|w_{0}\right|^{2}+n_{2}\left|v_{0}\right|^{2}\right)$ and the quantity $\epsilon=(1-u) \sqrt{u} \sin \left(\phi_{2}-2 \phi_{1}\right)+C u+D u^{2}$. Here $\phi_{1}(z)$ and $\phi_{2}(z)$ are the phases of $w_{0}(z)$ and $v_{0}(z)$, respectively, and $u(z)=\frac{1}{2} \eta_{0} n_{2}\left|v_{0}(z)\right|^{2} / I_{0}$ is the fraction of average intensity $I_{0}$ in the average $\mathrm{SH}$. The parameters are $D=\frac{3}{2} \gamma_{1} \sqrt{J} / \rho_{1}$ and $C=-\frac{1}{2} \beta /\left(\rho_{1} \sqrt{J}\right)-\frac{4}{3} D$, where $I_{0}=\frac{1}{2} \eta_{0} n_{2} J$. In the physical regime, where the polynomial $f(u)=-D^{2} u^{4}+(1-2 C D) u^{3}-\left(2+C^{2}-2 \epsilon D\right) u^{2}+$ $(1+2 \epsilon C) u-\epsilon^{2}$ has four real roots $u_{0} \leq u_{1} \leq 1 \leq u_{2} \leq u_{3}$, we solve Eqs. (4) by quadrature and find the average SH intensity

$$
u(z)=\left[u_{3} \operatorname{sn}^{2}(r z \mid m)+u_{0} p\right] /\left[\operatorname{sn}^{2}(r z \mid m)+p\right],
$$

where $p=\left(u_{3}-u_{1}\right) /\left(u_{1}-u_{0}\right), m p=\left(u_{3}-u_{2}\right) /\left(u_{2}-u_{0}\right)$, and $r=\frac{3}{2}\left|\gamma_{1}\right| J \sqrt{\left(u_{2}-u_{0}\right)\left(u_{3}-u_{1}\right)}$ are real positive parameters. The Jacobian Elliptic $\operatorname{sn}(z \mid m)$ function is periodic with period $4 \mathrm{~K}(\mathrm{~m})$, where $\mathrm{K}(\mathrm{m})$ is the complete elliptic integral of the first kind 15 . The solution (7) is thus periodic with the period $2 \mathrm{~K}(\mathrm{~m}) / r$ and becomes aperiodic when $u_{1}=u_{2}=1(m=1)$, in which case $100 \%$ transfer of power to the $\mathrm{SH}$ is predicted by the average model. From $f(1)=0$ we obtain the separatrix $C+D=\epsilon$.

The solution (7) was found earlier forno seeding of the average $\mathrm{SH}$, i.e. with $u(0)=u_{0}=\epsilon=0$ 10. In this case $I=I_{0}$ to lowest order and the separatrix $C+D=0$ corresponds to the physical intensity $I=I_{\mathrm{s}}^{0}=\frac{1}{2} \eta_{0} n_{2} J_{\mathrm{s}}^{0}$, where $J_{\mathrm{s}}^{0}=-\beta / \gamma_{1}$. It was further proven 10 that when the separatrix is crossed the phase-shift of the FW over a period changed by exactly $\pi$, a result that is also valid for our general solution (7). However, it was never investigated whether $I_{\mathrm{s}}^{0}=-\frac{1}{2} \eta_{0} n_{2} \beta / \gamma_{1}$ was an accurate prediction of the separatrix intensity in the physical system (112).

As a typical sample we consider bulk $\mathrm{LiNbO}_{3}$, a FW wavelength of $1.064 \mu \mathrm{m}, d_{\text {eff }}=30 \mathrm{pm} / \mathrm{V}, n_{1}=2.2$, and $n_{2}=2.23$, which gives the coherence length $L_{c}=8.9 \mu \mathrm{m}$. In Fig. 1 1 we show the nonlinear phase-shift of the FW versus the physical intensity $I$ as predicted by the unseeded average model (45) with $u(0)=v_{0}(0)=0$ and found by numerical integration of the corresponding physical system (1-2) with initial condition determined from Eqs. (3) and (6). With the domain length $L_{d}=8.8 \mu \mathrm{m}$ the predicted separatrix intensity $I_{\mathrm{s}}^{0}=231 \mathrm{GW} / \mathrm{cm}^{2}$ is $26 \mathrm{GW} / \mathrm{cm}^{2}$ too high compared to what is actually found in the physical system (11-2). This inaccuracy has not been observed before and is mainly due to the average $\mathrm{SH}$ being initially zero, which violates the requirement that the harmonics are small compared to the average field.

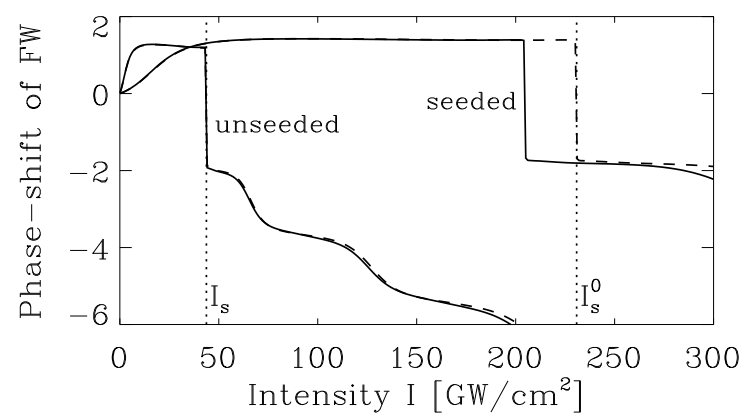

Fig. 1. Nonlinear phase-shift of the FW versus intensity $I$ for bulk $\mathrm{LiNbO}_{3}$, as predicted by the averaged model (4-5) (dashed) and found numerically from the physical Eqs. (112) (solid). The QPM domain length is $L_{d}=8.8 \mu \mathrm{m}$ and the crystal length is $L=351 \mu \mathrm{m}(174 \mu \mathrm{m})$ for unseeded (seeded) SHG. The vertical dotted lines mark the separatrices $I_{\mathrm{s}}$ and $I_{\mathrm{s}}^{0}$.

At this point we stress that no seeding in the average model (4.5) implies a seeding of the physical $S H$, as given by the transformations (3) and (6), i.e. $v_{0}(0)=0$ gives $E_{1}(0)=w_{0}(0)$ and $E_{2}(0)=-i\left(\sigma \rho_{2} / \kappa\right) w_{0}^{2}(0)$. The experimentally relevant setup is unseeded SHG with $E_{2}(0)=0$, for which Fig. 11 shows that the physical phase versus intensity curve is indistinguishable from the prediction of the average model ( $v_{0}$ is now seeded and thus no assumptions are violated). The physical separatrix intensity for unseeded $\mathrm{SHG}$ is $44 \mathrm{GW} / \mathrm{cm}^{2}$, i.e., 5.3 times lower than the prediction $I_{\mathrm{s}}^{0}$ of the unseeded averaged model. Note that the averaged model without the cubic terms would not predict any separatrix.

From Eqs. (3) and (6) with $E_{2}(0)=0$ we find that $v_{0}(0)=i\left(\rho_{2} / \kappa\right) x^{2}$ where $x=w_{0}(0)$ is real and $E_{1}(0)=$ $x+\left(\rho_{1} \rho_{2} / \kappa^{2}\right) x^{3}$. Then $\epsilon=n_{2} \rho_{2} \sqrt{J} /\left(n_{1} \kappa\right)$ to lowest order and thus $J_{\mathrm{s}}=-\left(1-8 / \pi^{2}\right) \beta / \gamma_{1}$. This gives the physical separatrix intensity for unseeded $S H G$

$$
I_{\mathrm{s}}=-\frac{\eta_{0} n_{2}}{2}\left(1-\frac{8}{\pi^{2}}\right) \frac{\beta}{\gamma_{1}}
$$

since again $I=I_{0}$ to lowest order. For $L_{d}=8.8 \mu \mathrm{m}$ this gives $I_{\mathrm{s}}=44 \mathrm{GW} / \mathrm{cm}^{2}$, which is exactly the numerically found value (see Fig. 11). Our numerical simulations confirm this accuracy for all values of the domain length satisfying $L_{d} \sim L_{c} \ll L$ and also for negative $\Delta k$.

A first requirement for the QPM-induced separatrix to be relevant for switching purposes is that the intensity $I_{\mathrm{S}}$ 
is low, which requires the ratio $\beta / \gamma_{1}$ to be small, i.e. the effective mismatch must be small (but nonzero) and the induced cubic nonlinearity strong. We will not discuss more appropriate materials than $\mathrm{LiNh}_{3} \mathrm{H}_{3}$, but we note that the average model is very general 6 , allowing $\gamma_{1}$ to be significantly increased by, e.g., modulating the QPM grating 9 or by a strong dc-value of the grating

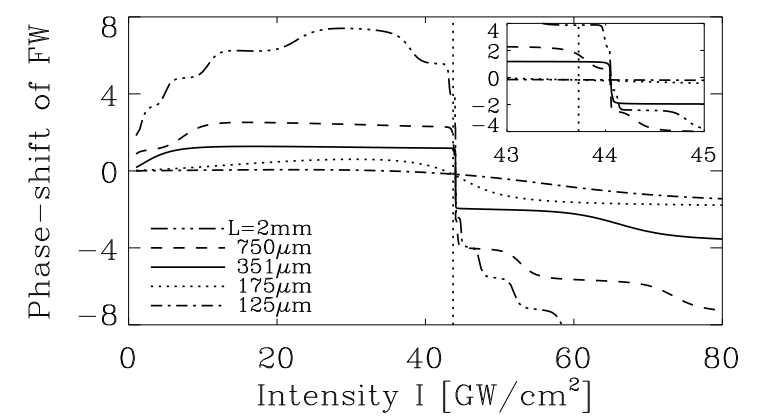

Fig. 2. Nonlinear phase-shift of the FW versus intensity $I$ for unseeded SHG in bulk $\mathrm{LiNbO}_{3}$. The QPM domain length is $L_{d}=8.8 \mu \mathrm{m}$. The crystal length is given in the figure. The vertical dotted line marks the separatrix $I_{\mathrm{s}}=44 \mathrm{GW} / \mathrm{cm}^{2}$.

So far the induced separatrix has not been observed in experiments. The reason could be: (i) The intensity was too low; (ii) the induced and inherent self-phase modulation (SPM) terms eliminate each other - conventional $\chi^{(2)}$ materials are self-focusing and have normal dispersion and thus the inherent SPM coefficient is positive, while the induced SPM coefficient $\left(-\gamma_{2}\right)$ is negative; (iii) the crystal length was inappropriate. In Fig. 2 we illustrate the effect of the crystal length. Clearly, the separatrix becomes more and more "hidden" in the overall variation due to the quadratic nonlinearity, and the flat plateaus on each side become narrower, as $L$ is increased from the optimal value around $351 \mu \mathrm{m}$. When $L$ is too short the separatrix is lost.
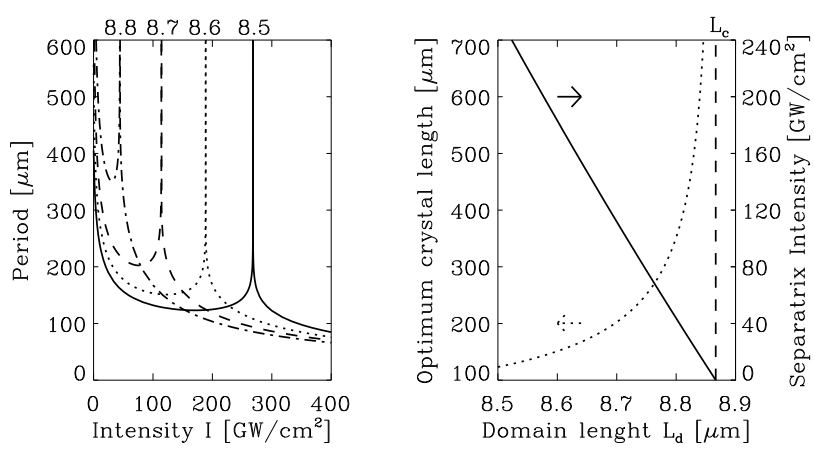

Fig. 3. Left: Period versus intensity $I$ for unseeded SHG in bulk QPM $\mathrm{LiNbO}_{3}$ with the QPM domain length given in microns at the curves. Right: Optimal crystal length and separatrix intensity $I_{\mathrm{s}}$ versus domain length.

In Fig. 3 we show the period $2 K(m) / r$ of the solu- tion (7) versus intensity. The minimum period between $I=0$ and the separatrix $I=I_{\mathrm{s}}$ provides a good measure of the optimal crystal length observed in Fig. 2, for which the $\pi$-shift due to the separatrix is the clearest and the flat phase versus intensity plateaus on either side are the broadest. From Fig. 3 we see how this optimal length increases as the domain length approaches the coherence length $8.9 \mu \mathrm{m}$, i.e. as exact effective phase-matching is approached. At the same time the separatrix intensity decreases.

Thus it is desirable to work close to, but not exactly at, exact effective phase-matching in order to reduce the holding intensity in the switching process and keep a reasonable crystal length. Clearly the resolution in the photolithographic process could be an issue if the separatrix is to be measured and used.

The research is supported by the Danish Technical Research Council through Talent Grant No. 5600-00-0355.

1. N.R. Belashenkov, S.V. Gagarskii, M.V. Inochkin, Opt. Spektrosk. 66 (1989) 1383 [Opt. Spectrosc. 66 (1989) 806].

2. R. DeSalvo, D.J. Hagan, M. Sheik-Bahae, G. Stegeman, E.W. Van Stryland, H. Vanherzeele, Opt. Lett. 17, 28 (1992).

3. For a review see G. Stegeman, D.J. Hagan, L. Torner, Opt. Quantum Electron. 28, 1691 (1996).

4. J.A. Armstrong, N. Bloembergen, J. Ducuing, P.S. Pershan, Phys. Rev. 127, 1918 (1962).

5. M.M. Fejer, in Beam Shaping and Control with Nonlinear Optics, F. Kajzar and R. Reinisch, eds. (Plenum, New York, 1998) pp. 375-406.

6. A. Saher Helmy, D.C. Hutchings, T.C. Kleckner, J.H. Marsh, A.C. Bryce, J.M. Arnold, C.R. Stanley, J.S. Aitchison, C.T.A. Brown, K. Moutzouris, M. Ebrahimzadeh, Opt. Lett. 25, 1370 (2000).

7. C. Balslev Clausen, O. Bang, Y.S. Kivshar, Phys. Rev. Lett. 78 (1997) 4749.

8. J.F. Corney, O. Bang, "Solitons in quadratic nonlinear photonic crystals", LANL, nlin.PS/0007042.

9. O. Bang, C. Balslev Clausen, P.L. Christiansen, and L. Torner, Opt. Lett. 24, 1413 (1999).

10. A. Kobyakov, F. Lederer, O. Bang, Y.S. Kivshar, Opt. Lett. 23 (1998) 506.

11. W. Choe, P.P. Banerjee, F.C. Caimi, J. Opt. Soc. Am. B 8, 1013 (1991).

12. L.S. Telegin, A.S. Chirkin, Kvantovaya Elektron. 9, 2086 (1982) [Sov. J. Quantum Electron. 12, 1354 (1982)].

13. Y. Zhao, G. Town, M. Sceats, Opt. Commun. 115, 129 (1995).

14. C.R. Menyuk, R. Schiek, L. Torner, J. Opt. Soc. Am. B 11, 2434 (1994). O. Bang, ibid. 14, 51 (1997).

15. M. Abramowitz, I.A. Stegun, Handbook of Mathematical Functions, 9th ed. (Dover, New York, 1972). 